The Journal of $\mathbf{N}_{\text {onlinear }} \mathbf{S}$ ciences and Applications http://www.tjnsa.com

\title{
COUPLED FIXED POINTS OF SET VALUED MAPPINGS IN PARTIALLY ORDERED METRIC SPACES
}

\author{
ISMAT BEG ${ }^{1 *}$ AND ASMA RASHID BUTT ${ }^{2}$
}

\begin{abstract}
Let $(X, \preceq)$ be a partially ordered set and $d$ be a metric on $X$ such that $(X, d)$ is a complete metric space. Let $F: X \times X \rightsquigarrow X$ be a mixed monotone set valued mapping. We obtain sufficient conditions for the existence of a coupled fixed point of $F$.
\end{abstract}

\section{INTRODUCTION AND PRELIMINARIES}

Let $(X, d)$ be a complete metric space and $C B(X)$ be the class of all nonempty closed and bounded subsets of $X$. For $A, B \in C B(X)$, let

$$
D(A, B):=\max \left\{\sup _{b \in B} d(b, A), \sup _{a \in A} d(a, B)\right\},
$$

where

$$
d(a, B):=\inf _{b \in B} d(a, b),
$$

$D$ is said to be a Hausdorff metric induced by $d$.

Banach's contraction principle [14, Theorem 2.1] assures the existence of a unique fixed point if a self mapping on a complete metric space $X$ is a contraction. Nadler [16] in 1969, proved a set valued extension of the Banach's contraction principle in complete metric spaces. He proved the following result:

Theorem 1.1. [16] Let $(X, d)$ be a complete metric space and $F: X \rightsquigarrow X$ be a set valued mapping such that $F(x)$ is a nonempty closed bounded subset of $X$.

Date: Received: 21 Oct. 2010.

*Corresponding author

(c) 2010 N.A.G.

2000 Mathematics Subject Classification. Primary 47H10; Secondary 47H04; $47 \mathrm{H} 07$.

Key words and phrases. Coupled fixed point, partially ordered set, metric space, set valued mapping. 
If there exists $\kappa \in(0,1)$ such that

$$
D(F(x), F(y)) \leq \kappa d(x, y), \text { for all } x, y \in X,
$$

then $F$ has a fixed point in $X$.

Afterward many fixed point theorems have been proved by various authors as generalization of the Nadler's theorem where the nature of contractive mapping is weakened along with some additional requirements, see for instance [2, 8, 9, 10, 15, 22, 24, 26] and references cited there in. Mixed monotone mappings have been discussed by different authors due to its wide applications. The theory of mixed monotone single valued mappings in ordered Banach spaces has been investigated in [1, 6, 11, 25, 27]. Ran and Reurings [23] established the existence of unique fixed point for the monotone single valued mapping in partially ordered metric spaces. Their result was further extended in [3, 4, 5, 7, 12, 13, 17, 18, 19, 20, 21]. Вy following the trend initiated by Ran and Reurings, Bhaskar and Lakshmikantham in [7] studied the existence and uniqueness of a coupled fixed point in partially ordered metric space with assumption that the single valued mapping satisfy the weaker contraction condition. The aim of this paper is to obtain results on the existence of a coupled fixed point for set valued mappings in partially ordered metric spaces by following the technique of Bhaskar and Lakshmikantham [7]. Let $F: X \times X \rightsquigarrow X$ be a set valued mapping i.e., $X \ni(x, y) \mapsto F(x, y)$ is a subset of $X$.

Definition 1.2. A point $(x, y) \in X \times X$ is said to be a coupled fixed point of the set valued mapping $F$ if $x \in F(x, y)$ and $y \in F(y, x)$.

Definition 1.3. A point $x \in X$ is said to be a fixed point of the set valued mapping $F$ if $x \in F(x, x)$.

Definition 1.4. A partial order relation is a binary relation $\preceq$ on $X$ which satisfies the following conditions:

i) $x \preceq x$ (reflexivity),

ii) if $x \preceq y$ and $y \preceq x$ then $x=y$ (antisymmetry),

iii) if $x \preceq y$ and $y \preceq z$ then $x \preceq z$ (transitivity),

for all $x, y$ and $z$ in $X$.

A set with a partial order $\preceq$ is called a partially ordered set .

Definition 1.5. Let $(X, \preceq)$ be a partially ordered set and $x, y \in X$. Elements $x$ and $y$ are said to be comparable elements of $X$ if either $x \preceq y$ or $y \preceq x$.

Definition 1.6. Let $X$ be a partially ordered set and $F: X \times X \rightsquigarrow X$ be a set valued mapping. $F$ is said to be a mixed monotone mapping if $F$ is orderpreserving in $x$ and order-reversing in $y$ i.e., $x_{1} \preceq x_{2}, y_{2} \preceq y_{1}, x_{i}, y_{i} \in X(i=1,2)$ implies for all $u_{1} \in F\left(x_{1}, y_{1}\right)$ there exists $u_{2} \in F\left(x_{2}, y_{2}\right)$ such that $u_{1} \preceq u_{2}$ and for all $v_{1} \in F\left(y_{1}, x_{1}\right)$ there exists $v_{2} \in F\left(y_{2}, x_{2}\right)$ such that $v_{2} \preceq v_{1}$.

Lemma 1.7. [16] If $A, B \in C B(X)$ with $D(A, B)<\epsilon$ then for each $a \in A$ there exists an element $b \in B$ such that $d(a, b)<\epsilon$.

Lemma 1.8. [16] Let $\left\{A_{n}\right\}$ be a sequence in $C B(X)$ and $\lim _{n \rightarrow \infty} D\left(A_{n}, A\right)=0$ for $A \in C B(X)$. If $x_{n} \in A_{n}$ and $\lim _{n \rightarrow \infty} d\left(x_{n}, x\right)=0$, then $x \in A$. 


\section{Coupled Fixed Points}

Let $(X, \preceq)$ be a partially ordered set and $d$ be a metric on $X$ such that $(X, d)$ is a complete metric space. We define the partial order on the product space $X \times X$ as:

for $(u, v),(x, y) \in X \times X, \quad(u, v) \preceq(x, y)$ if and only if $u \preceq x, v \succeq y$. The product metric on $X \times X$ is defined as:

$$
d\left(\left(x_{1}, y_{1}\right),\left(x_{2}, y_{2}\right)\right):=d\left(x_{1}, x_{2}\right)+d\left(y_{1}, y_{2}\right), \text { for all } x_{i}, y_{i} \in X(i=1,2) .
$$

For notational convenience we use the same symbol $d$ for the product metric as well as for the metric on $X$.

We begin with the following theorem that gives the existence of a coupled fixed point (not necessarily unique) in partially ordered metric spaces for the set valued mappings.

Theorem 2.1. Let $F: X \times X \rightsquigarrow X$ be a set valued mapping with non empty closed bounded values satisfying:

(1) There exists $\kappa \in(0,1)$ with

$$
D(F(x, y), F(u, v)) \leq \frac{\kappa}{2} d((x, y),(u, v)) \text {, for all }(x, y) \succeq(u, v) .
$$

(2) If $x_{1} \preceq x_{2}, y_{2} \preceq y_{1}, x_{i}, y_{i} \in X(i=1,2)$ then for all $u_{1} \in F\left(x_{1}, y_{1}\right)$ there exists $u_{2} \in F\left(x_{2}, y_{2}\right)$ with $u_{1} \preceq u_{2}$ and for all $v_{1} \in F\left(y_{1}, x_{1}\right)$ there exists $v_{2} \in F\left(y_{2}, x_{2}\right)$ with $v_{2} \preceq v_{1}$ provided $d\left(\left(u_{1}, v_{1}\right),\left(u_{2}, v_{2}\right)\right)<1$.

(3) There exists $x_{0}, y_{0} \in \bar{X}$, and some $x_{1} \in F\left(x_{0}, y_{0}\right), y_{1} \in F\left(y_{0}, x_{0}\right)$ with $x_{0} \preceq x_{1}, y_{0} \succeq y_{1}$ such that $d\left(\left(x_{0}, y_{0}\right),\left(x_{1}, y_{1}\right)\right)<1-\kappa$, where $\kappa \in(0,1)$.

(4) If a nondecreasing sequence $x_{n} \rightarrow x$ in $X$ then $x_{n} \preceq x$, for all $n$ and if a nonincreasing sequence $y_{n} \rightarrow y$ in $X$ then $y_{n} \succeq y$, for all $n$.

Then $F$ has a coupled fixed point.

Proof. Let $x_{0}, y_{0} \in X$ then by assumption 3 there exists $x_{1} \in F\left(x_{0}, y_{0}\right), y_{1} \in$ $F\left(y_{0}, x_{0}\right)$ with $x_{0} \preceq x_{1}, y_{0} \succeq y_{1}$ such that

$$
d\left(\left(x_{0}, y_{0}\right),\left(x_{1}, y_{1}\right)\right)<1-\kappa .
$$

Since $\left(x_{0}, y_{0}\right) \preceq\left(x_{1}, y_{1}\right)$ by using assumption 1 and inequality 1 , we have

$$
D\left(F\left(x_{0}, y_{0}\right), F\left(x_{1}, y_{1}\right)\right) \leq \frac{\kappa}{2} d\left(\left(x_{0}, y_{0}\right),\left(x_{1}, y_{1}\right)\right)<\frac{\kappa}{2}(1-\kappa) .
$$

and similarly

$$
D\left(F\left(y_{0}, x_{0}\right), F\left(y_{1}, x_{1}\right)\right) \leq \frac{\kappa}{2}(1-\kappa) .
$$

Using assumption 2 and Lemma 1.7, we have the existence of $x_{2} \in F\left(x_{1}, y_{1}\right), y_{2} \in$ $F\left(y_{1}, x_{1}\right)$ with $x_{1} \preceq x_{2}$ and $y_{1} \succeq y_{2}$ such that

$$
d\left(x_{1}, x_{2}\right) \leq \frac{\kappa}{2}(1-\kappa) .
$$

and

$$
d\left(y_{1}, y_{2}\right) \leq \frac{\kappa}{2}(1-\kappa)
$$


From inequalities 2 and 3,

$$
d\left(\left(x_{1}, y_{1}\right),\left(x_{2}, y_{2}\right)\right) \leq \kappa(1-\kappa) .
$$

Again by assumption 1 and inequality 4 we have,

$$
D\left(F\left(x_{1}, y_{1}\right), F\left(x_{2}, y_{2}\right)\right) \leq \frac{\kappa^{2}}{2}(1-\kappa)
$$

and

$$
D\left(F\left(y_{1}, x_{1}\right), F\left(y_{2}, x_{2}\right)\right) \leq \frac{\kappa^{2}}{2}(1-\kappa)
$$

Further from Lemma 1.7 and assumption 2 we have the existence of $x_{3} \in F\left(x_{2}, y_{2}\right)$, $y_{3} \in F\left(y_{2}, x_{2}\right)$ with $x_{2} \preceq x_{3}, y_{2} \succeq y_{3}$ such that

and

$$
d\left(x_{2}, x_{3}\right) \leq \frac{\kappa^{2}}{2}(1-\kappa) \text {. }
$$

It follows that,

$$
d\left(y_{2}, y_{3}\right) \leq \frac{\kappa^{2}}{2}(1-\kappa)
$$

$$
d\left(\left(x_{2}, y_{2}\right),\left(x_{3}, y_{3}\right)\right) \leq \kappa^{2}(1-\kappa) .
$$

Continuing in this way we obtain $x_{n+1} \in F\left(x_{n}, y_{n}\right), y_{n+1} \in F\left(y_{n}, x_{n}\right)$ with $x_{n} \preceq$ $x_{n+1}, y_{n} \succeq y_{n+1}$ such that,

and

$$
d\left(x_{n}, x_{n+1}\right) \leq \frac{\kappa^{n}}{2}(1-\kappa)
$$

Thus

$$
d\left(y_{n}, y_{n+1}\right) \leq \frac{\kappa^{n}}{2}(1-\kappa)
$$

$$
d\left(\left(x_{n}, y_{n}\right),\left(x_{n+1}, y_{n+1}\right)\right) \leq \kappa^{n}(1-\kappa) .
$$

Next we will show that $\left(x_{n}\right)$ is a Cauchy sequence in $X$. Let $m>n$. Then

$$
\begin{aligned}
d\left(x_{n}, x_{m}\right) & \leq d\left(x_{n}, x_{n+1}\right)+d\left(x_{n+1}, x_{n+2}\right)+d\left(x_{n+2}, x_{n+3}\right)+\ldots+d\left(x_{m-1}, x_{m}\right) \\
& \leq\left[\kappa^{n}+\kappa^{n+1}+\kappa^{n+2}+\ldots+\kappa^{m-1}\right] \frac{(1-\kappa)}{2} \\
& =\kappa^{n}\left[1+\kappa+\kappa^{2} \ldots+\kappa^{m-n-1}\right] \frac{(1-\kappa)}{2} \\
& =\kappa^{n}\left[\frac{1-\kappa^{m-n}}{1-\kappa}\right] \frac{(1-\kappa)}{2} \\
& =\frac{\kappa^{n}}{2}\left(1-\kappa^{m-n}\right)<\frac{\kappa^{n}}{2},
\end{aligned}
$$

because $\kappa \in(0,1), 1-\kappa^{m-n}<1$.

Therefore $d\left(x_{n}, x_{m}\right) \rightarrow 0$ as $n \rightarrow \infty$ implies that $\left(x_{n}\right)$ is a Cauchy sequence and hence converges to some point (say) $x$ in the complete metric space $X$.

Similarly, we can show that $\left(y_{n}\right)$ is also a Cauchy sequence in $X$. Since $X$ is a complete metric space, there exists $x, y \in X$ such that $x_{n} \rightarrow x$ and $y_{n} \rightarrow y$ as $n \rightarrow \infty$. Finally we have to show that $x \in F(x, y)$ and $y \in F(y, x)$.

Since $x_{n}$ is a nondecreasing sequence and $y_{n}$ is a nonincreasing sequence in $X$ such that $x_{n} \rightarrow x$ and $y_{n} \rightarrow y$ therefore we have $x_{n} \preceq x$ and $y_{n} \succeq y$ for all $n$.

From assumption 1, it follows that

$$
D\left(F\left(x_{n}, y_{n}\right), F(x, y)\right) \leq \kappa d\left(\left(x_{n}, y_{n}\right),(x, y)\right) \rightarrow 0 .
$$


Now because $x_{n+1} \in F\left(x_{n}, y_{n}\right)$ and $\lim _{n \rightarrow \infty} d\left(x_{n+1}, x\right)=0$, it follows by using Lemma 1.8 that $x \in F(x, y)$. Again by 1 ,

$$
D\left(F\left(y_{n}, x_{n}\right), F(y, x)\right) \leq \kappa d\left(\left(y_{n}, x_{n}\right),(y, x)\right) \rightarrow 0 .
$$

Since $y_{n+1} \in F\left(y_{n}, x_{n}\right)$ and $\lim _{n \rightarrow \infty} d\left(y_{n+1}, y\right)=0$, it follows by using Lemma 1.8 that $y \in F(y, x)$. Hence $(x, y)$ is a coupled fixed point of the set valued mapping $F$.

The contraction condition given by Nadler [16] is stonger than the contraction condition used in our Theorems 2.1. Also Theorem 2.1 generalize the result of Bhaskar and Lakshmikantham [7]

Corollary 2.2. Let $X$ be a partially ordered set and $d$ be a metric on $X$ such that $(X, d)$ is a complete metric space. Let $f: X \times X \rightarrow X$ be a single valued mapping satisfying

(1) there exists $\kappa \in(0,1)$ with

$$
D(f(x, y), f(u, v)) \leq \frac{\kappa}{2}[d(x, u)+(y, v)], \text { for all }(x, y) \succeq(u, v) .
$$

(2) $f$ is a mixed monotone mapping.

(3) there exists $x_{0}, y_{0} \in X$ with $x_{0} \preceq f\left(x_{0}, y_{0}\right)=x_{1}$ and $y_{0} \succeq f\left(y_{0}, x_{0}\right)=$ $y_{1}$ (say).

(4) if a nondecreasing sequence $x_{n} \rightarrow x$ in $X$, then $x_{n} \preceq x$, for all $n$ and if a nonincreasing sequence $y_{n} \rightarrow y$ in $X$ then $y_{n} \succeq y$, for all $n$.

Then $f$ has a coupled fixed point.

Remark 2.3. If in assumption 4 of Theorem 2.1, $x$ and $y$ are comparable then $x=y$ and $x \in F(x, x)$ :

Let $x \preceq y$ or $x \succeq y$. Then

$$
D\left(F(x, y), F(y, x) \leq \frac{\kappa}{2}[d(x, y)+d(y, x)]=\kappa d(x, y) .\right.
$$

Because $x \in F(x, y)$ and $y \in F(y, x)$ by Lemma 1.8, $d(x, y) \leq \kappa d(x, y)$. This implies that $d(x, y)=0$, since $\kappa \in(0,1)$. Thus $x=y$ and $x \in F(x, x)$.

Example 2.4. Let $X=\{-0.1,0,0.1\}$ be a subset of $R$ with usual order. Let $d$ be a metric on $X$ defined by $d(x, y)=|x-y|$ for each $x, y \in X$ so that $(X, d)$ is a complete metric space. Consider $X \times X$ with partial order $(u, v) \preceq(x, y)$ if and only if $u \preceq x, v \succeq y$. Now the product metric $d$ is given by

$$
d\left(\left(x_{1}, y_{1}\right),\left(x_{2}, y_{2}\right)\right):=\left|x_{1}-x_{2}\right|+\left|y_{1}-y_{2}\right| .
$$

Let $F: X \times X \rightsquigarrow X$ be a set valued mapping defined as:

$$
F(x, y)=\left\{\begin{array}{c}
\emptyset \text { if } x=y \\
\{0,0.1\} \text { if } x>y . \\
X \text { if } x<y
\end{array}\right.
$$

Now since $(0,0.1) \preceq(0.1,0)$, therefore $D(F(0.1,0), F(0,0.1))=0$ and $d((0.1,0),(0,0.1))=0.2$.

Also $(0,0.1) \preceq(0.1,-0.1), D(F(0,0.1), F(0.1,-0.1))=0.1$ and $d((0,0.1),(0.1,-0.1))=0.3$. 
Similarly for other comparable elements contraction condition is satisfied with $\kappa=0.5$.

All the conditions of Theorem 2.1 satisfied and $F$ has a coupled fixed point at $(0,0.1)$.

\section{REFERENCES}

[1] I. Beg, Random fixed points of increasing compact maps, Archivum Mathematicum 37 (2001), 329-332.

[2] I. Beg and A. Azam, Fixed points of asymptotically regular multivalued mappings, J. Austral. Math. Soc. (Series-A) 53(3) (1992), 313-326.

[3] I. Beg and A.R. Butt, Fixed point for set valued mappings satisfying an implicit relation in partially ordered metric spaces, Nonlinear Anal. 71 (2009), 3699-3704.

[4] I. Beg and A. R. Butt, Fixed points for weakly compatible mappings satisfying an implicit relation in partially ordered metric spaces, Carpathian J. Math. 25 (2009), 1-12.

[5] I. Beg and A.R. Butt: Common fixed point for generalized set valued contractions satisfying an implicit relation in partially ordered metric spaces, Mathematical Communications, 15 (2010), 65-76.

[6] I. Beg, A. Latif, R. Ali and A. Azam, Coupled fixed points of mixed monotone operators on probabilistic Banach spaces, Archivum Mathematicum 37 (2001), 1-8.

[7] T.G. Bhaskar and V. Lakshmikantham, Fixed point theorems in partially ordered metric spaces and applications, Nonlinear Anal., 65 (2006), 1379-1393.

[8] P.Z. Daffer, Fixed points of generalized contractive multivalued mappings, J. Math. Anal. Appl., 192 (1995), 655-666.

[9] P.Z. Daffer, H. Kaneko and W. Li, On a conjecture of S. Reich, Proc. Amer. Math. Soc., 124 (1996), 3159-3162.

[10] Y. Feng and S. Liu, Fixed point theorems for multivalued contractive mappings and multivaled Caristi type mappings, J. Math. Anal. Appl., 317 (2006), 103-112.

[11] D. Guo and V. Lakshmikantham, Coupled fixed points of nonlinear operators with applications, Nonlinear. Anal., 11 (1987), 623-632.

[12] J. Harjani, K. Sadarangani, Fixed point theorems for weakly contractive mappings in partially ordered sets, Nonlinear Anal. 71 (2009), 3403-3410.

[13] J. Harjani, K. Sadarangani, Generalized contractions in partially ordered mtric spaces and applications to ordinary differential equations, Nonlinear Anal. (2009), doi:10.1016/j.na.2009.08.003(in press).

[14] W.A. Kirk and K.Goebel, Topics in Metric Fixed Point Theory, Cambridge University Press, Cambridge 1990.

[15] D. Klim and D.Wardowski, Fixed point theorems for set-valued contractions in complete metric spaces, J. Math. Anal. Appl., 334 (2007), 132-139.

[16] S.B. Nadler, Multivalued contraction mappings, Pacific J. Math., 30 (1969), 475-488.

[17] J.J. Nieto, R.L. Pouso and R. Rodríguez-López, Fixed point theorems in ordered abstract spaces, Proc. Amer. Math. Soc., 135 (2007), 2505-2517.

[18] J.J. Nieto and R. Rodríguez-López, Contractive mapping theorms in partially ordered sets and applications to ordinary differential equations, Order, 22 (2005), 223-239.

[19] J.J. Nieto and R. Rodríguez-López, Existence and uniqueness of fixed point in partially ordered sets and applications to ordinary differential equations, Acta. Math. Sinica, (English Ser.) 23 (2007), 2205-2212.

[20] D. O'Regan and A. Petrusel, Fixed point theorems for generalized contractions in ordered metric spaces, J. Math. Anal. Appl., 341 (2008), 1241-1252.

[21] A. Petrusel and I.A. Rus, Fixed point theorems in ordered L-spaces, Proc. Amer. Math. Soc., 134 (2005), 411-418.

[22] C.Y. Qing, On a fixed point problem of Reich, Proc. Amer. Math. Soc., 124 (1996), 30853088. 
[23] A.C.M. Ran and M.C.B. Reurings, A fixed point theorm in partially ordered sets and some applications to matrix equations, Proc. Amer. Math. Soc., 132 (2004), 1435-1443.

[24] S. Reich, Fixed points of contractive functions, Boll. Unione. Mat. Ital., (4) (1972), 26-42.

[25] Y. Wu, New fixed point theorems and applications of mixed monotone operator, J. Math. Anal. Appl., 341 (2008), 883-893.

[26] E. Zeidler, Nonlinear Functional Analysis and its Applications I: Fixed point Theorems, Springer Verlag, New York 1985.

[27] Z. Zhitao, New fixed point theorems of mixed monotone operators and applications, J. Math. Anal. Appl., 204 (1996), 307-319.

1 Centre for Advanced Studies in Mathematics, Lahore University of Management SCIEnCES, 54792-Lahore, PAKISTAN.

E-mail address: ibeg@lums.edu.pk

${ }^{2}$ Centre for Advanced Studies in Mathematics, Lahore University of ManAGEMEnt SCIENCES, 54792-LAhore, PAKISTAN.

E-mail address: asmar@lums.edu.pk 